\title{
Avaliação neuropsicológica de idosos em investigação para demência*
}

\section{Neuropsychological evaluation of elderly people investigated for dementia}

\section{Evaluación neuropsicológica de ancianos en investigación para la demencia}

\section{Recebido: 22/01/2018 \\ Aprovado: 18/08/2018 \\ Publicado: 05/11/2018}

\author{
Sabrina Martins Barroso ${ }^{1}$ \\ Raphaela Campos de Sousa ${ }^{2}$
}

O objetivo deste estudo foi realizar a avaliação neuropsicológica de idosos em investigação diagnóstica para quadros demenciais na cidade de Uberaba, MG. Foram avaliados 35 idosos, com média de idades de 72,57 anos, sendo a maior parte do sexo feminino e baixa escolaridade. Utilizou-se os testes: Mini-Exame do Estado Mental, Teste do Relógio, Caminhada Cronometrada, Nove Pinos, Bateria CERAD e questionário complementar. Os resultados dos testes foi dicotomizada entre "normal" e "déficit". Foram conduzidas análises descritivas e correlacionais. Os resultados mostraram capacidade preservada de memória imediata e de reconhecimento, fluência verbal, preensão e controle dos membros superiores para a maioria dos avaliados. Déficits foram identificados quanto a orientação têmporo-espacial, memória de longo prazo, praxias, nomeação, habilidades visuoespaciais e de planejamento e controle dos membros inferiores. Houve associação entre desempenhos em diferentes testes. A pesquisa mostrou relevância da avaliação neuropsicológica integrada à investigação de quadros demenciais.

Descritores: Idoso; Neuropsicologia; Função executiva; Avaliação da deficiência.

The objective of this study was to perform a neuropsychological evaluation of elderly people undergoing diagnostic investigation for dementia in the city of Uberaba/MG, Brazil. Thirty-five elderly individuals, with a mean age of 72.57 years, were studied. Most were females and with low schooling. The tests were: MiniMental State Examination, Clock-Drawing Test, Timed Walking Test, Nine-Hole Peg Test, CERAD Battery and a complementary questionnaire. The test results were dichotomized between "normal" and "deficit". Descriptive and correlational analyzes were conducted. The results showed preserved ability of immediate memory and memory, verbal fluency, grip and control of the upper limbs for the majority of the evaluated participants. Deficits were identified regarding temporal and spatial orientation, long-term memory, praxis, naming, visuospatial and planning skills, and control of the lower limbs. There was an association between performance in different tests. The research showed the relevance of the neuropsychological evaluation integrated to the investigation of demential pictures.

Descriptors: Aged; Neuropsychology; Executive function; Disability evaluation.

El objetivo de este estudio fue realizar la evaluación neuropsicológica de ancianos en investigación diagnóstica para cuadros demenciales en la ciudad de Uberaba, MG, Brasil. Se evaluaron 35 ancianos, con promedio de edades de 72,57 años, siendo la mayor parte del sexo femenino y baja escolaridad. Se utilizaron las pruebas: Mini-Examen del Estado Mental, Prueba del Reloj, Caminata Cronometrada, Nueve Pines, Batería CERAD y cuestionario complementario. Los resultados de las pruebas fueron dicotomizadas entre "normal" y "déficit". Se realizaron análisis descriptivos y correlacionales. Los resultados mostraron capacidad preservada de memoria inmediata y de reconocimiento, fluencia verbal, asimiento y control de los miembros superiores para la mayoría de los evaluados. Los déficits fueron identificados como la orientación témporoespacial, memoria de largo plazo, praxis, nombramiento, habilidades visu-espaciales y de planificación y control de los miembros inferiores. Hubo asociación entre desempeños en diferentes pruebas. La investigación mostró relevancia de la evaluación neuropsicológica integrada a la investigación de cuadros demenciales.

Descriptores: Anciano; Neuropsicología; Función ejecutiva; Evaluación de la discapacidad.

1. Psicóloga. Especialista em Desenvolvimento Humano. Mestre em Psicologia. Doutora em Saúde Pública. Professora Adjunta do Departamento de Psicologia e Coordenadora do Programa de Pós-Graduação em Psicologia (PPGP) da Universidade Federal do Triângulo Mineiro (UFTM), Uberaba, MG, Brasil. ORCID: 0000-0003-1759-9681 E-mail: smb.uftm@gmail.com

2. Psicóloga. Mestranda em Psicologia pelo PPGP/UFTM. Professora o Curso de Psicologia da Universidade de Uberaba (UNIUBE), Uberaba, MG, Brasil.. ORCID: 0000-0002-3142-3044E-mail: rapha1618.rc@gmail.com

* Este estudo teve financiamento da Fundação de Amparo a Pesquisa de Minas Gerais (FAPEMIG). 


\section{INTRODUÇÃO}

$\mathbf{E}$ m 2015 a população idosa brasileira era de $14,3 \%{ }^{1}$, havendo projeções que em 2070 o país será o sexto país com maior número de idosos no mundo representando mais de $35 \%$ do total do país ${ }^{1}$. Esse aumento da população idosa tem gerado maior interesse de pesquisadores e profissionais sobre a temática do envelhecimento ${ }^{2}$.

0 envelhecimento gera alterações físicas, cognitivas e comportamentais, derivadas do envelhecimento celular e de experiências de vida. Algumas alterações são percebidas de forma positiva, ligadas ao aumento da maturidade e de conhecimentos. Outras envolvem a perda da velocidade de processamento de informações e flexibilidade cognitiva, ou se relacionam com transtornos neurocognitivos ${ }^{3,4}$.

Um dos aspectos que vem recebendo destaque dentro das investigações envolve os transtornos neurodegenerativos, que possuem múltiplas etiologias e representam um grupo heterogêneo de patologias. De acordo com o DSM-5, para o diagnóstico de transtorno neurocognitivo é preciso que exista comprometimento da memória em conjunto com déficit em pelo menos um dos seguintes domínios cognitivos: linguagem, praxia, gnosia ou funções executivas ${ }^{4}$. Essas patologias prejudicam a independência funcional e criam a dependência de um cuidador externo para os idosos ${ }^{5-8}$.

A Doença de Alzheimer é a maior responsável pelo surgimento de quadros demenciais entre idosos, respondendo mundialmente por $70 \%$ dos casos diagnosticados de transtorno neurocognitivo nessa população. Outras causas frequentes são a Demência Vascular, Demência de Corpos de Lewy e Demência Frontotemporal ${ }^{6}$.

Como os transtornos neurocognitivos geram grandes limitações e respondem melhor a tratamento quando descobertos em etapas mais precoces, identificá-los em seu período pródomo torna-se relevante ${ }^{9}$. Caso haja uma identificação precoce, tratamentos medicamentosos e não medicamentosos, tais como a reabilitação cognitiva, podem ser iniciados e contribuir para retardar o surgimento ou evitar o aparecimento dos sintomas $^{10}$, melhorando a qualidade de vida de idosos e cuidadores ${ }^{9}$.

A avaliação neuropsicológica é usada como uma ferramenta para identificação de déficits cognitivos e diagnóstico demencial precoce. Serve, ainda, para avaliação de aspectos funcionais do desempenho, que podem dificultar a realização de tarefas cotidianas ${ }^{2}$. A realização de avaliações dessa natureza é dificultada em cidades do interior pela ausência de profissionais treinados para executá-las, o que limita as informações dessa natureza em diversas regiões.

Não há informações sobre as características neuropsicológicas dos idosos da região do Triângulo Mineiro até 0 momento, o que impede a busca de semelhanças e especificidades em relação a outras regiões. Além disso, compromete o desenvolvimento de planos de intervenção adaptados à realidade local, o que é indicado como importante para o sucesso de qualquer intervenção cognitiva4. Considerando a importância de identificar precocemente os déficits cognitivos em idosos, o presente estudo tem como objetivo realizar a avaliação neuropsicológica de idosos em investigação diagnóstica para quadros demenciais na cidade de Uberaba, MG.

\section{MÉTODO}

Estudo transversal, descritivo e correlacional, baseado em metodologia quantitativa de interpretação dos dados.

Participaram do estudo 35 idosos em investigação diagnóstica para déficit cognitivo leve, Mal de Parkinson, Doença de Alzheimer ou Demência Vascular, no Ambulatório de Neurologia do Hospital de Clínicas da Universidade Federal do Triângulo Mineiro (HC-UFTM).

Os participantes representavam todos os idosos em investigação diagnóstica para as patologias elencadas durante o segundo semestre de 2016. Foram considerados critérios de inclusão, ter 60 anos ou mais, frequentar o HC-UFTM e estar em investigação diagnóstica para demência de qualquer natureza, déficit cognitivo leve ou Mal de Parkinson. Não houve restrição quanto 
ao sexo, presença de dificuldade física ou comorbidades físicas.

Em termos de instrumentos de avaliação se utilizou-se: a entrevista, O Mini Exame do Estado Mental (MEEM), o teste do relógio, a caminhada cronometrada de nove pinos, a Bateria CERAD e provas clínicas.

A entrevista usada tinha roteiro semiestruturado, desenvolvido para a presente avaliação que abordou as condições de saúde, histórico de vida, sintomatologia, dados sociodemográficos e queixas. O MEEM é considerado um conjunto de provas clínicas que avalia as funções básicas de orientação espacial e temporal, memória e linguagem, com pontos de corte estabelecidos em função da escolaridade. São considerados 13 pontos para analfabetos, 18 para pessoas com 1 a 7 anos de estudo e 26 pontos para pessoas com mais de 8 anos de estudo ${ }^{11-12}$.

Já o Teste do Relógio que se usou foi a versão adotada de correção foi desenvolvida por Sunderland ${ }^{13} \mathrm{e}$ validada para população idosa no Brasil ${ }^{14}$. Avalia percepção visuoespacial, capacidade de planejamento e memória operacional, por meio da evocação e reprodução de um relógio analógico. Atribuise uma pontuação de 0 a 10 pontos, sendo pontuações até 5 pontos indicativas de déficits e entre 6 e 10 pontos desempenho normal.

A Caminhada Cronometrada e Nove Pinos - Tarefas adaptadas e validadas para o Brasil 15 , avaliam a capacidade de controle dos membros superiores e inferiores. A caminhada é realizada por uma reta de 7,62 metros, com duração de até 10 segundos. 0 controle dos membros superiores é verificado pela repetição de uma tarefa de retirada e colocação de nove pinos de madeira de uma base, separadamente com cada mão. 0 controle dos membros superiores é considerado normal se as tarefas são desempenhadas com tempo total de até 150 segundos.

A Bateria CERAD - desenvolvida por Morris ${ }^{16}$, foi adaptada para o Brasil em 200117, sendo composta pelos testes de Fluência Verbal - Animais, Nomeação de Boston, listas de palavras (recordação imediata, tardia e reconhecimento) e teste de habilidade construtiva. Cada subteste possui um ponto de corte específico, definidos em função da escolaridade, para diferenciar resultados normais e déficits.

Nas Provas Clínicas ou provas de avaliação de comportamento voluntário, considerou-se: controle inibitório, preensão, instruções conflitantes e encadeamento de pensamento.

O projeto foi aprovado pelo Comitê de Ética em Pesquisa da Universidade Federal do Triângulo Mineiro (Protocolo 2663/2010). Após tal aprovação os idosos foram convidados a participar voluntariamente do estudo. Foram convidados todos os idosos em investigação diagnóstica que frequentaram o HC-UFTM ao longo do segundo semestre de 2016.

O convite foi feito pessoalmente, por um integrante da equipe de pesquisa, diretamente ao idoso, durante o tempo de sala de espera para atendimento neurológico. Nesse momento os idosos que concordaram com a pesquisa agendavam com 0 pesquisador um horário para coleta de dados presencial.

A coleta dos dados ocorreu em dois encontros, com duração média de duas horas cada. Tais encontros foram realizados nas dependências do HC-UFTM ou na casa dos idosos, conforme preferência dos avaliados, em horários previamente agendados. Não havia idosos curatelados na amostra, por isso os próprios participantes foram convidados e os que aceitaram participar assinaram o Termo de Consentimento Livre e Esclarecido. Após a correção dos instrumentos a equipe sistematizou os resultados em laudos individuais que foram devolvidos para os idosos e, com sua autorização, disponibilizados para os neurologistas que acompanhavam os casos.

Foram realizadas análises descritivas de porcentagem, tendência central e dispersão para caracterização da amostra e das condições cognitivas e físicas dos idosos. Realizou-se, também, análise de correlação de Spearman, considerando significância de 5\% entre o desempenho dos idosos e sua escolaridade. 
A correlação com a escolaridade se baseou na influência dos anos de estudo sobre a escolarização, demonstrada em trabalhos anteriores ${ }^{18}$ e a correlação entre os diferentes desempenhos foi feita considerando achados sobre a interligação dos aspectos cognitivos ${ }^{19}$.

\section{RESULTADOS}

Os idosos participantes (35) do estudo foram predominantemente mulheres (71,4\%), com idade variando entre 61 e 86 anos e média de $72,57 \pm 7,30$ anos, analfabeta ou com baixa escolaridade $(77,2 \%)$ conforme a Tabela 1.

Tabela 1. Idosos de acordo com sexo e escolaridade. Uberaba, MG, 2016.

\begin{tabular}{lrcc}
\hline \multicolumn{1}{c}{ Variáveis } & $\mathbf{n}$ & $\mathbf{\%}$ \\
\hline Sexo & Feminino & 25 & 71,4 \\
& Masculino & 10 & 28,6 \\
& & & \\
& Analfabetos & 15 & 42,9 \\
& $\mathbf{0 - 3}$ anos estudo & 12 & 34,3 \\
& $\mathbf{4 - 8}$ anos estudo & 03 & 8,6 \\
& $\mathbf{9}$ ou + anos estudo & 05 & 14,3 \\
\hline
\end{tabular}

No controle motor dos membros superiores, observou-se que 92,3\% dos participantes apresentou controle para a mão dominante e $91,7 \%$ para a mão nãodominante no teste nove pinos. 0 comportamento de preensão foi considerado normal para a maioria da amostra $(88,6 \%)$ e $64,6 \%$ dos avaliados conseguiam reproduzir os movimentos de mão realizados pelo avaliador. Nas habilidades avaliadas pelas provas clínicas, a maior parte dos idosos tinha habilidade de evocação de praxia preservada $(54,3 \%)$, mas mostrava déficit na praxia construtiva $(66,7 \%)$ de acordo com a Tabela 2.

Sobre o controle dos membros inferiores, o teste de marcha mostrou uma média de 10,39 \pm 6,72 segundos, o que é um tempo superior ao esperado para indicar preservação dessa função. Em termos relativos, apenas $26,7 \%$ dos respondentes mostraram capacidade preservada de controle dos membros inferiores. Nos casos de déficit (73,3\%), 20\% apresentavam ausência de coordenação entre mãos e pés, $13,3 \%$ mostraram problemas para caminhar em linha reta e 6,7\% apresentou marcha vacilante (Tabela 2).

$\mathrm{Na}$ avaliação cognitiva, a maioria dos participantes mostrou déficit no MEEM
(51,4\%), ficando a média da pontuação em $19,54 \pm 7,54$ pontos. Déficits na capacidade de encadeamento de pensamento, necessária para contar histórias com início, meio e fim de forma coerente, foi observada em 54,2\% (Tabela 2).

No teste de instruções conflitantes, $57,6 \%$ da amostra copiou as batidas do examinador em uma superfície sólida mais de duas vezes, o que indica déficit na capacidade de sensibilidade ao meio. Quanto à fluência verbal, observou-se resultado dentro do esperado para $62,9 \%$ dos casos. Mas houve déficit na capacidade de nomeação para a maioria dos avaliados $(80,0 \%)$ (Tabela 2$)$.

Quanto a capacidade de planejamento, percepção visuoespacial e memória operacional, por meio do teste do relógio, verificou-se déficit em 59,3\% dos avaliados. Mas ao fornecer uma pista externa para execução da tarefa, por meio do modelo desenhado pelo avaliador, o número de pessoas com desempenho normal atingiu 65,5\%. Quanto à recordação observou-se capacidade preservada para memória imediata em 57,1\% dos respondentes e para reconhecimento em $60 \%$. Entretanto, houve déficit para a maioria dos avaliados na capacidade de recordação tardia $(68,6 \%)$ (Tabela 2). 
Tabela 2. Idosos conforme testes neuropsicológicos e relação entre normal e deficit. Uberaba, MG, 2016.

\begin{tabular}{|c|c|c|}
\hline Variáveis & $\mathbf{n}$ & $\%$ \\
\hline \multicolumn{3}{|l|}{ Mini-mental } \\
\hline Normal & 17 & 48,6 \\
\hline Déficit & 18 & 51,4 \\
\hline \multicolumn{3}{|l|}{ Recordação Imediata } \\
\hline Normal & 20 & 57,1 \\
\hline Déficit & 15 & 42,9 \\
\hline \multicolumn{3}{|l|}{ Recordação Tardia } \\
\hline Normal & 11 & 31,4 \\
\hline Déficit & 24 & 68,6 \\
\hline \multicolumn{3}{|l|}{ Memória de Reconhecimento } \\
\hline Normal & 21 & 60,0 \\
\hline Déficit & 14 & 40,0 \\
\hline \multicolumn{3}{|l|}{ Relógio Evocação } \\
\hline Normal & 11 & 31,4 \\
\hline Déficit & 16 & 45,7 \\
\hline Não Avaliados & 08 & 22,9 \\
\hline \multicolumn{3}{|l|}{ Relógio Cópia } \\
\hline Normal & 19 & 54,3 \\
\hline Déficit & 10 & 28,6 \\
\hline Não Avaliados & 06 & 17,1 \\
\hline \multicolumn{3}{|l|}{ Marcha } \\
\hline Normal & 22 & 62,9 \\
\hline Déficit & 08 & 22,9 \\
\hline Não Avaliados & 05 & 14,3 \\
\hline \multicolumn{3}{|l|}{ Fluência Verbal } \\
\hline Normal & 22 & 62,9 \\
\hline Déficit & 13 & 37,1 \\
\hline \multicolumn{3}{|l|}{ Praxia Construtiva } \\
\hline Normal & 10 & 28,6 \\
\hline Déficit & 23 & 66,7 \\
\hline Não Avaliados & 02 & 5,7 \\
\hline \multicolumn{3}{|l|}{ Evocação da Praxia } \\
\hline Normal & 19 & 54,3 \\
\hline Déficit & 16 & 45,7 \\
\hline \multicolumn{3}{|l|}{ Pensamento Encadeado } \\
\hline Normal & 11 & 31,4 \\
\hline Déficit & 24 & 68,6 \\
\hline \multicolumn{3}{|l|}{ Nomeação } \\
\hline Normal & 03 & 20,0 \\
\hline Déficit & 32 & 80,0 \\
\hline \multicolumn{3}{|l|}{ Nove Pinos - mão dominante } \\
\hline Normal & 24 & 68,6 \\
\hline Déficit & 02 & 5,7 \\
\hline Não Avaliados & 09 & 25,7 \\
\hline \multicolumn{3}{|l|}{ Nove Pinos - mão não-dominante } \\
\hline Normal & 22 & 62,9 \\
\hline Déficit & 02 & 5,7 \\
\hline Não Avaliados & 11 & 31,4 \\
\hline
\end{tabular}

A análise das relações entre as variáveis mostrou que as caraterísticas individuais interferiram com o desempenho dos idosos nos testes cognitivos. Os participantes com maior escolaridade tiveram melhor desempenho na tarefa de evocação do teste do relógio (rho $=0,43 ; \mathrm{p} \leq 0,05$ ), melhor desempenho no teste de nomeação (rho $=0$, 52; $\mathrm{p} \leq 0,001$ ) e na evocação da praxia construtiva (rho $=0,51 ; \mathrm{p} \leq 0,001$ ). Além disso, a classificação de capacidade cognitiva normal no MEEM mostrou associação com 
preservação da capacidade de recordação imediata (rho $=0,49 ; \mathrm{p} \leq 0,001$ ), memória de reconhecimento (rho $=0,44 ; \mathrm{p} \leq 0,001$ ), fluência verbal (rho $=0,39 ; \mathrm{p} \leq 0,05)$, controle inibitório (rho $=0,38 ; \mathrm{p} \leq 0,05$ ) $\mathrm{e}$ comportamento de preensão (rho $=-0,34 ; \mathrm{p} \leq$ $0,05)$ (Tabela 3).

Os resultados também mostraram a relação entre as capacidades cognitivas dos idosos. Os idosos que tiveram melhor desempenho na tarefa de evocação do teste do relógio também mostraram melhor desempenho na tarefa de cópia do teste do relógio (rho $=0,58 ; \mathrm{p} \leq 0,001$ ), na recordação imediata (rho $=0,53 ; \mathrm{p} \leq 0,001$ ) e tardia (rho $=0,42 ; \mathrm{p} \leq 0,05)$. As pessoas que obtiveram classificação normal na tarefa de cópia do teste do relógio também se saíram melhor na recordação tardia (rho $=0,44 ; \mathrm{p} \leq 0,05$ ), recordação imediata (rho $=0,42 ; \mathrm{p} \leq 0,05$ ), fluência verbal (rho $=0,45 ; p \leq 0,05)$, evocação da praxia (rho $=0,48 ; \mathrm{p} \leq 0,05$ ), praxia construtiva (rho $=0,52 ; \mathrm{p} \leq 0,001$ ) $\mathrm{e}$ nomeação (rho = 0,37; $\mathrm{p} \leq 0,001$ ) (Tabela 3).

$\mathrm{Na}$ capacidade de linguagem as pessoas com classificação na capacidade de nomeação também mostraram classificação normal nos testes de fluência verbal (rho $=0,38 ; \mathrm{p} \leq 0,05$ ), evocação da praxia (rho $=0,68 ; \mathrm{p} \leq 0,001$ ), instruções conflitantes ( $r h o=0,36 ; p \leq 0,05$ ), controle inibitório (rho $=0,50 ; p \leq 0,001$ ) e pensamento encadeado ( $r h o=0,51 ; p \leq 0,05$ ). A classificação de normalidade no teste de fluência verbal esteve associada à normalidade no teste de instruções conflitantes (rho $=0,54 ; \mathrm{p} \leq 0,001$ ), controle inibitório (rho $=0,45 ; \mathrm{p} \leq 0,001$ ), encadeamento de pensamento (rho $=0,59 ; \mathrm{p}$ $\leq 0,001$ ), evocação da praxia (rho $=0,45$; $\mathrm{p} \leq$ $0,05)$ e controle dos membros superiores ao realizar o teste com sua mão dominante (rho $=0,39 ; \mathrm{p} \leq 0,05)$ (Tabela 3).

Na relação entre os aspectos as pessoas com indicação de capacidade de recordação imediata preservada mostraram ter a capacidade de recordação tardia (rho $=0,46$; $\mathrm{p} \leq 0,05$ ) e reconhecimento (rho $=0,47$; $0,05)$ também preservada (Tabela 3 ).

A memória também mostrou relação com outras funções. Houve correlação entre a classificação normal no teste de recordação imediata e a capacidade de evocação da praxia (rho = 0,53; $\mathrm{p}<0,05$ ), nomeação (rho = 0,43; $\mathrm{p} \leq 0,001$ ), fluência verbal (rho $=0,65 ; \mathrm{p} \leq$ 0,001 ), encadeamento de pensamento ( $\mathrm{rho}=$ 0,$51 ; \mathrm{p} \leq 0,001$ ) e controle inibitório (rho = 0,$40 ; p \leq 0,05$ ) (Tabela 3).

A memória de longo prazo mostrou relação com a evocação da praxia (rho $=0,51$; $\mathrm{p} \leq 0,05$ ) e fluência verbal (rho $=0,39 ; \mathrm{p} \leq$ $0,05)$, capacidade para lidar com instruções conflitantes (rho $=0,44 ; \mathrm{p} \leq 0,001$ ) $\mathrm{e}$ capacidade de preensão (rho $=0,43 ; \mathrm{p} \leq$ 0,001 ), fora as relações já indicadas com o desempenho no teste do relógio (Tabela 3). 
Tabela 3. Idosos conforme correlações entre os desempenhos cognitivos.Uberaba, MG, 2016.

\begin{tabular}{|c|c|c|c|c|c|c|c|c|c|c|c|c|c|c|}
\hline & Escola & $\begin{array}{l}\operatorname{Rec}^{1} . \\
\text { Imediata }\end{array}$ & $\begin{array}{l}\text { Rec } \\
\text { Tardia }\end{array}$ & $\begin{array}{l}\text { Reconhe- } \\
\text { cimento }\end{array}$ & $\begin{array}{l}\text { Relógio } \\
\text { Evoc. }^{2}\end{array}$ & $\begin{array}{l}\text { Relógio } \\
\text { Cópia }\end{array}$ & MEEM & Marcha & Fluência & Praxia & $\begin{array}{l}\text { Evoc. } \\
\text { Praxia }\end{array}$ & $\begin{array}{l}\text { Pensa- } \\
\text { mento }\end{array}$ & Nome $^{3}$ & $\begin{array}{l}9 \text { Pinos } \\
\text { domi- } \\
\text { nante }\end{array}$ \\
\hline Escola & 1 & & & & & & & & & & & & & \\
\hline $\begin{array}{l}\text { Rec. } \\
\text { Imediata }\end{array}$ & $-0,30$ & 1 & & & & & & & & & & & & \\
\hline Rec. Tardia & $-0,18$ & $0,46^{* *}$ & 1 & & & & & & & & & & & \\
\hline $\begin{array}{l}\text { Reconhe- } \\
\text { cimento }\end{array}$ & $-0,08$ & $0,47^{* *}$ & 0,17 & 1 & & & & & & & & & & \\
\hline $\begin{array}{l}\text { Relógio } \\
\text { Evocação }\end{array}$ & $-0,43^{*}$ & $0,53^{* *}$ & $0,45^{*}$ & 0,10 & 1 & & & & & & & & & \\
\hline $\begin{array}{l}\text { Relógio } \\
\text { Cópia }\end{array}$ & $-0,33$ & $0,42^{*}$ & $0,44^{*}$ & 0,03 & $0,58^{* *}$ & 1 & & & & & & & & \\
\hline MEEM & $-0,12$ & $0,49 * *$ & 0,20 & $0,44^{*}$ & 0,28 & 0,17 & 1 & & & & & & & \\
\hline Marcha & $-0,18$ & $-0,03$ & $-0,01$ & 0,21 & $-0,15$ & $-0,06$ & 0,81 & 1 & & & & & & \\
\hline Fluência & $-0,18$ & $0,64^{* *}$ & $0,39 *$ & $0,33^{*}$ & $-0,37$ & $0,45^{*}$ & $0,39 *$ & 0,21 & 1 & & & & & \\
\hline Praxia & $-0,34$ & 0,29 & 0,33 & 0,12 & 0,37 & $0,52^{* *}$ & 0,11 & 0,13 & 0,22 & 1 & & & & \\
\hline Evocação & - & 0,24 & 0,25 & $-0,04$ & 0,23 & 0,36 & $-0,14$ & 0,00 & 0,12 & $-0,37^{*}$ & 1 & & & \\
\hline Praxia & $0,51^{* *}$ & & & & & & & & & & & & & \\
\hline Pensamento & 0,27 & $-0,53^{* *}$ & $-0,23$ & $-0,19$ & $-0,11$ & $-0,43$ & $-0,19$ & $-0,12$ & $-0,59 * *$ & $-0,41^{*}$ & $-0,19$ & 1 & & \\
\hline Nomeação & - & $-0,43^{* *}$ & 0,27 & 0,26 & 0,28 & $0,37^{*}$ & 0,22 & 0,30 & $0,38^{*}$ & $0,46^{*}$ & 0,31 & $-0,51^{*}$ & 1 & \\
\hline $\begin{array}{l}9 \text { Pinos } \\
\text { dominante }\end{array}$ & $-0,03$ & 0,31 & $-0,15$ & 0,36 & 0,16 & 0,26 & 0,31 & 0,17 & $0,39 *$ & 0,12 & $-0,06$ & $-0,59 * *$ & 0,14 & 1 \\
\hline $\begin{array}{l}9 \text { Pinos não- } \\
\text { dominante }\end{array}$ & $-0,26$ & 0,35 & 0,17 & 0,10 & 0,24 & 0,38 & 0,05 & $-0,17$ & 0,13 & 0,19 & 0,23 & $-0,19$ & 0,15 & $0,69^{* *}$ \\
\hline
\end{tabular}

Notas: ${ }^{1} \operatorname{Rec}=$ Recordação; ${ }^{2}$ Evoc $=$ Evocação; ${ }^{3}$ Nome $=$ Nomeação. ${ }^{* *} p<0,001 ;{ }^{*} p \leq 0,0$ 


\section{DISCUSSÃO}

Os resultados do presente estudo identificaram problemas na capacidade de memorização e em outras habilidades cognitivas nos idosos avaliados. Mostraram, ainda, relações entre os desempenhos em diferentes testes. Essas correlações ilustram como os diferentes aspectos cognitivos interagem e precisam ser considerados de forma integrada para qualquer avaliação diagnóstica ${ }^{3,6,18}$.

Estudo anterior ${ }^{20}$ investigou as funções executivas nos idosos e demonstrou que há relação entre os componentes cognitivos, particularmente entre memória de trabalho, velocidade de processamento de informações, memória de longo prazo e inteligência fluida.

Os principais déficits de memória foram observados na memória de longo prazo, o que não é o esperado nos quadros demenciais iniciais. A memória de curto prazo em geral é afetada nas pessoas com transtornos neurocognitivos, para, posteriormente, serem percebidos déficits na memória de longo prazo $^{3,5}$.

Os idosos avaliados não possuíam diagnóstico estabelecido. Esse indício serviu para levantar novas hipóteses diagnósticas para explicar seus sintomas autorreferidos e mensurados. $\mathrm{Na}$ apresentação desses resultados à equipe médica, houve solicitação de exames que não haviam sido inicialmente pensados, tais como nível de vitamina $\mathrm{B} 12$ e D, avaliação de hipotireoidismo e encaminhamento de alguns idosos para avaliação de estado emocional.

Essas avaliações são importantes, pois a carência dessas vitaminas, condições clínicas ligadas à tireoide e quadros não identificados de depressão podem afetar o desempenho da memória4.Déficits na memória são prérequisitos para diagnóstico de qualquer síndrome demencial, mas a identificação de outras disfunções cognitivas e do nível de comprometimento pode auxiliar no diagnóstico diferencial e na fase em que a patologia se encontra 4 . A identificação precoce dos quadros demenciais aumenta a efetividade da intervenção e contribui para manutenção da independência funcional por maior tempo ${ }^{9}$.

A triagem cognitiva inicial foi feita por meio do MEEM. Esse é um teste de triagem com sensibilidade para identificar apenas casos moderados ou graves de deterioração cognitiva $^{21}$ e os idosos avaliados eram relativamente jovens (média de 72,57 anos), mas vindos de uma população em investigação para síndrome demencial.

Essa particularidade pode auxiliar a entender porque a maioria dos respondentes mostrou comprometimento nessa avaliação. Acrescenta-se aí, o fato de que a maioria eram analfabetos ou possuíam baixa escolaridade, fator esse que se mostrou associado ao pior desempenho nos testes. Estudos anteriores ${ }^{7-}$ 8,22 , já haviam indicado que a maior escolaridade influencia para a manutenção das funções cognitivas em idosos, assim como, que a escolarização precoce, contribui de forma importante para a criação de uma reserva cognitiva, que minimiza o impacto das lesões cerebrais ${ }^{7,8,23,24}$.

Ao avaliar a linguagem, a fluência mostrou-se preservada, mas houve déficit na capacidade de nomeação. Esses resultados mostram a importância de atentar para a redução do vocabulário ao avaliar casos de suspeita de quadro demencial e seu possível impacto na estruturação do pensamento. A correlação observada entre os resultados de nomeação e encadeamento de pensamento corroboram os achados de outro estudo ${ }^{5}$, da qual déficits de linguagem em pessoas com demência leve e moderada afetam os processos de pensamento.

No teste do relógio como medida de percepção visuespacial, memória operacional e capacidade de planejamento, o desempenho mostrou que a capacidade para evocar espontaneamente essas funções já estava prejudicada, mas ainda podia ser acessada por meio de pistas ambientais. Esse padrão já havia sido descrito em outro estudo ${ }^{14}$. Por sua vez, o teste do relógio associou-se com o desempenho na linguagem e memória, indicando, novamente, a importância de considerar as habilidades cognitivas de forma integrada. 
A comparação com o desempenho de idosos de outras regiões mostrou um perfil similar de características sociodemográficas e de desempenho cognitivo, mostrando um perfil parecido dos idosos residentes em Uberaba com outros investigados.

\section{CONCLUSÃO}

Os resultados observados nos idosos em investigação diagnóstica para síndromes demenciais mostraram capacidades cognitivas preservadas, entre elas a fluência verbal, percepção visuoespacial e memória operacional com auxílio de pistas externas, memória imediata e de reconhecimento, além do controle dos membros superiores.

Contudo, também foram observados déficits que podem contribuir para refinamento diagnóstico, tais como: dificuldades para orientação temporal e espacial, déficits na memória de longo prazo, praxias, nomeação, percepção visuoespacial e memória de trabalho sem auxílio, sensibilidade às interferências ambientais e controle dos membros inferiores. 0 desempenho cognitivo não se mostrou independente, havendo associação significativa entre as diferentes tarefas avaliadas.

0 presente estudo apresentou limitações quanto à amostra e utilizou apenas testes de rastreio cognitivo. Essas condições, adotadas por se tratar de um estudo inicial da temática na região limitam a capacidade de generalização dos achados e a identificação de déficits mais sutis.

Apesar disto, houve relevância ao se investigar as funções cognitivas de forma integrada e contribuição da avaliação neuropsicológica para diagnósticos demenciais.

Estudos futuros, com amostras maiores e testes mais sensíveis poderão melhorar a compreensão sobre as condições cognitivas dos idosos da cidade de Uberaba e contribuir para diagnósticos neurológicos ou psiquiátricos mais acurados e precoces.

\section{REFERÊNCIAS}

1. Instituto Brasileiro de Geografia e Estatística. Síntese de indicadores sociais: uma análise das condições de vida da população brasileira: 2016 [Internet]. Rio de Janeiro: IBGE; 2016 [citado em 19 dez 2017]. Disponível em: https://biblioteca.ibge.gov.br/visualizacao/livr os/liv98965.pdf

2. Veras R. Envelhecimento populacional contemporâneo: demandas, desafios e inovações. Rev Saúde Pública. 2009; 43(3):54854.

3. Hamdan AC, Pereira APAM, Sá Riechi TIJ. Avaliação e reabilitação neuropsicológica: desenvolvimento histórico e perspectivas atuais. Interação Psicol. 2011; 15(Esp):47-58.

4. Associação Americana de Psiquiatria. DSM-5: manual diagnóstico e estatístico de transtornos mentais. Porto Alegre: Artmed; 2014.

5. Koehler C, Gindri G, Bós AJG, Mancopes R. Alterações de linguagem em pacientes idosos portadores de demência avaliados com a Bateria MAC. Rev Soc Bras Fonoaudiol. 2012; 17(1):15-22.

6. Nascimento ERN, Barbosa MA, Brasil VV, Sousa ALL, Amaral GF, Jácomo PJ. Qualidade de vida de quem cuida de portadores de demência com corpos Lewy. J Bras Psiquiatr. 2013; 62(2):144-52.

7. Burlá C, Camarano AA, Kanso S, Fernandes D, Nunes R. Panorama prospectivo das demências no Brasil: um enfoque demográfico. Ciênc Saúde Coletiva. 2013; 18(10):2949-56.

8. Sobral M, Pestana MH, Paúl C. Cognitive reserve and the severity of Alzheimer's disease. Arq Neuropsiquiatr. [Internet]. 2015 [citado em: 22 dez 2017]; 73(6):480-6. doi: http://dx.doi.org/10.1590/0004-

282X20150044

9. Sobral M, Paúl C. Reserva cognitiva, envelhecimento e demências. RevE-Psi. [Internet]. 2015 [citado em: $22 \mathrm{dez}$ 2017]; 5(1):113-34. Disponível em: https://revistaepsi.com/wpcontent/uploads/artigos/2015/Ano5-

Volume1-Artigo6.pdf

10. Caixeta L, Peleja AC, Barros N. Doença de alzheimer: contribuições neuropsicológicas para o diagnóstico precoce. In: Caixeta L, Ferreira SB. Manual de neuropsicologia: dos princípios à reabilitação. Belo Horizonte: Atheneu; 2012. p. 235-250.

11. Gondim AS, Coelho Filho JM, Cavalcanti AA, Roriz Filho JS, Nogueira CB, Peixoto Junior AA, et al. Prevalence of functional cognitive impairment and associated factors in Brazilian community-dwelling older adults. Dement 
neuropsychol. [Internet]. 2017 [citado em 22 dez 2017]; 11(1):32-9. DOI: http://dx.doi.org/10.1590/1980-

57642016dn11-010006

12. Brucki SM, Campacci SR, Juliani Y. The minimental state examination in a general population: impact of educational status. Arq Neuropsiquiatr. [Internet]. 1994 [citado em: 22 dez 2017]; 52(1):1-7. DOI: http://dx.doi.org/10.1590/S0004282X1994000100001

13. Lourenço RA, Veras RP. Mini-exame do estado mental: características psicométricas em idosos ambulatoriais. Rev Saúde Pública. [Internet]. 2006 [citado em: 22 dez 2017]; 40(4):712-9.

http://dx.doi.org/10.1590/S0034-

89102006000500023

14. Sunderland T, Hill JL, Mellow AM, Lawlor BA, Gundersheimer J, Newhouse PA, et al. Clock drawing in Alzheimer's disease: a novel measure of dementia severity. J Am Geriatr Soc. [Internet]. 1989 [citado em: $22 \mathrm{dez}$ 2017]; 37(8):725-9.

http://dx.doi.org/10.1111/j.1532-

5415.1989.tb02233.x

15. Atalaia-Silva KC, Lourenço RA. Tradução, adaptação e validação de construto do Teste do Relógio aplicado entre idosos no Brasil. Rev Saúde Pública. [Internet]. 2008 [citado em 22 dez 2017]; 42(5):930-7. DOI: http://dx.doi.org/10.1590/S0034-

89102008000500020

16. Tilbery CP, Mendes MF, Thomaz RB, Oliveira BES, Kelian GL, Busch R, et al. Padronização da multiplesclerosis functional composite measure (MSFC) na população brasileira. Arq Neuropsiquiatr. [Internet]. 2005 [citado em: 22 dez 2017]; 63(1):127-32. DOI: http://dx.doi.org/10.1590/S0004-

282X2005000100023

17. Morris JC, Heyman A, Mohs RC, Hughes JP, van Belle G, Fillenbaum G, et al. The consortium to establish a registry for Alzheimer's disease (CERAD). Part I. Clinical and neuropsychological assessment of Alzheimer's disease. Neurology. 1989; 39(9):1159-65.

18. Steibel NM, Olchik MR, Yassuda MS, Finger G, Gomes I. Influence of age and educationon the River mead Behavioral Memory Test (RBMT) among healthy elderly. Dement Neuropsychol. [Internet]. 2016 [citado em: $19 \mathrm{dez}$ 2017];
10(1):26-30. Disponível em: http://www.scielo.br/pdf/dn/v10n1/1980-

5764-dn-10-01-00026.pdf

19. Mecca TP, Jana TA, Simões MR, Macedo EC.

Relação entre habilidades cognitivas nãoverbais e variáveis presentes no contexto educacional. Psicol Esc Educ. [Internet]. 2015 [citado em: 22 dez 2017]; 19(2):329-39. DOI: http://dx.doi.org/10.1590/21753539/2015/0192844

20. Bertolucci PHF, Okamoto IH, Brucki SMD, Siviero MO, Toniolo Neto J, Ramos LR. Applicability of the CERAD neuropsychological battery to brazilian elderly. Arq Neuropsiquiatr. [Internet]. 2001 [citado em: $22 \mathrm{dez}$ 2017]; 59(3A):532-6.

DOI:

http://dx.doi.org/10.1590/S0004-

282X2001000400009

21. Studart Neto A, Nitrini R. Subjective cognitive decline: the first clinical manifestation of Alzheimer's disease? Dement neuropsychol. [Internet]. 2016 [citado em $22 \mathrm{dez}$ 2017]; 10(3):170-7.

DOI:

http://dx.doi.org/10.1590/S1980-5764-

2016DN1003002

22. Mota MMPE, Banhato EFC, Silva KCA,

Cupertino APFB. Triagem cognitiva: comparações entre o mini-mental e o teste de trilhas. Estud Psicol. [Internet]. 2008 [citado em 22 dez 2017]; 25(3):353-9. DOI: http://dx.doi.org/10.1590/S0103-

166X2008000300004

23. Coelho CLM, Bastos CL, Camara FP, Landeira-Fernandes J. A influência do gênero e da escolaridade no diagnóstico de demência. Estud Psicol. [Internet]. 2010 [citado em $22 \mathrm{dez}$ 2017]; 27(4):449-56. DOI: http://dx.doi.org/10.1590/S0103-

166X2010000400003

24. Stern Y. Cognitive reserve: implications for assessment and intervention. Folia Phoniatr Logop. [Internet]. 2013 [citado em $22 \mathrm{dez}$ 2017]; 65(2):49-54. DOI: https://doi.org/10.1159/000353443

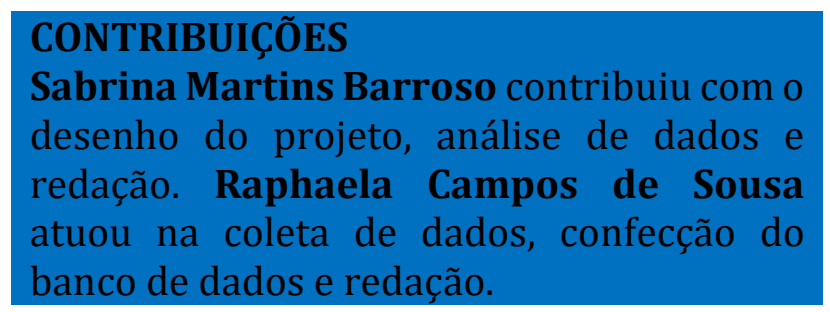


Como citar este artigo (Vancouver)

Barroso SM, Sousa RCS. Avaliação neuropsicológica de idosos em investigação para demência. REFACS [Internet]. 2018 [citado em: inserir dia, mês e ano de acesso]; 6(4):753763. Disponível em: inserir link de acesso. DOI: inserir link do DOI.

Como citar este artigo (ABNT)

BARROSO, S. M.; SOUSA, R. C. S. Avaliação neuropsicológica de idosos em investigação para demência. REFACS, Uberaba, MG, v. 6, n. 4, p. 753-763, 2018. Disponível em: <inserir link de acesso >. Acesso em: inserir dia, mês e ano de acesso. DOI: inserir link do DOI.

Como citar este artigo (APA)

Barroso, S.M \& Sousa, R.C.S. (2018). Avaliação neuropsicológica de idosos em investigação para demência. REFACS, 6(4), 753-763. Recuperado em: inserir dia, mês e ano de acesso de inserir link de acesso. DOI: inserir link do DOI. 